\title{
Improving limits of detection. Microdisc versus microcylinder electrodes.
}

\author{
Kristína Cinková, a Max Clark, ${ }^{\mathrm{b}}$ Stanislav V. Sokolov, ${ }^{\mathrm{b}}$ Christopher Batchelor-McAuley, ${ }^{\mathrm{b}}$ Lubomir Švorc, ${ }^{\mathrm{a}}$ Richard G. \\ Compton ${ }^{\text {b* }}$ \\ a Institute of Analytical Chemistry, Faculty of Chemical and Food Technology, Slovak University of Technology in Bratislava, \\ Radlinského 9, Bratislava, SK-812 37, Slovak Republic \\ b Department of Chemistry, Physical \& Theoretical Chemistry Laboratory, Oxford University, Oxford OX1 3QZ, United \\ Kingdom \\ * e-mail: richard.compton@chem.ox.ac.uk
}

Received:

Accepted:

\begin{abstract}
The specific apparent capacitance (Farads per unit geometric area) of commercial carbon microdisc electrodes and inhouse fabricated carbon microcylinders is studied through the use of cyclic voltammetry. Home-made microcylinder electrodes exhibit lower specific apparent capacitances and as a result facilitate lower limits of detection. We further demonstrate practically how the electroanalytical limit of the detection of solution phase trace oxygen is significantly improved using such microcylinder electrodes. Finally we show that after the degassing of a solution by nitrogen purging even in the most commonly encountered 'best' case scenarios approximately tens of $\mu \mathrm{M}$ of oxygen remain present in the electrochemical cell, unobservable by the commercial microdisc electrodes, but detectable with microcylinder electrodes, testifying to their analytical superiority.
\end{abstract}

Keywords: cyclic voltammetry, microdisc electrode, microwire electrode, apparent capacitance, limit of detection

DOI: $10.1002 /$ elan

\section{Introduction}

Electroanalysis usually requires measurement of the currents arising from the reduction or oxidation of redox active species. Ideally this capacitative charging of an electrode commonly sets a practical limit upon the smallest such Faradaic currents that are measurable using a given experimental system. This capacitance predominantly arises from the double layer charging of an electrode. The adsorption/desorption of specific ions [1-4] and the influence of the electronic structure of the material [5] can also contribute. Simplistically we can assume that the charging of the electrode can be modelled as an RC circuit [6]. Then in a cyclic voltammogram the capacitative current is the difference between the current measured on the forward and back scan thus comes from the charging of the electrode and is approximately equal to:

$i=2 A C v$.

Here $v$ is the experimental scan rate, the parameter $A$ is the electroactive area of the electrode and $C$ is the capacitance of the electrode $\left(\sim 20 \mu \mathrm{F} \mathrm{cm}^{-2}\right.$ in aqueous media) [6,7], where $C$ is assumed constant. Note that the electrode area is sensitive to the roughness of the electrode. In particular, A is approximately given by the geometric area of the electrode multiplied by a roughness factor. At a pragmatic level the magnitude of these background capacitative charging currents can be viewed as defining a practical limit of detection for a solution phase redox active species. This limit of detection can be taken to be the concentration of analyte where the signalto-background ratio is equal to unity. This is where the magnitude of the Faradaic current $\left(i_{F}\right)$ is equal to the capacitative electrode charging $\left(i_{C}\right)$. It should be commented that the measurement of smaller Faradaic currents below this limit is feasible but the analysis becomes more subjective and sensitive to the data analysis methodology employed [8].

On the basis of the above argument microelectrodes have been proposed for the detection of ultra-low concentrations of solution phase analytes [9]. Specifically for a microdisc electrode and measurements at low 
voltage scan rates then the diffusive mass-transport to the electrode is convergent. A high overpotentials the Faradaic current is found to be diffusion controlled steady-state given by $[6,10]$ :

$I_{s s}=4 n F D C r$.

Here $n$ is the number of electrons transferred, $F$ is the Faraday constant $\left(96485.3 \mathrm{C} \mathrm{mol}^{-1}\right), D$ is the diffusion coefficient of the analyte $\left(\mathrm{m}^{2} \mathrm{~s}^{-1}\right), C$ is the analyte concentration $\left(\mathrm{mol} \mathrm{m} \mathrm{m}^{-3}\right.$ ) and $r$ is the radius of the electrode. Ideally, the capacitance of the electrode is proportional to the electrode area if it arises from the double layer effects. The magnitude of the steady-state current varies linearly with the radius of the electrode. Thus as the size of the electrode decreases the practical electroanalytical limit of detection of a solution phase species is predicted to be proportional to the electrode radius. On this basis, for a microdisc electrode with a diameter of one micron the limit of detection is predicted to be of the order of $100 \mathrm{nM}$.

Such a low limit of detection of solution phase species is in reality challenging to realize. First, the detection of currents below tens of picoamperes becomes experimentally difficult. Generally, it is not practical for many real-world analytical applications. One route around this is to use an array of diffusionally independent electrodes. In this way the total current is substantially larger [11]. The use of microelectrode arrays has found widespread application [12]. However, avoiding the direct measurement of small currents comes at the expense of increasing the complexity of the electrode fabrication procedure. Moreover screen printed and gold microelectrode arrays do not lend themselves to being mechanically cleaned (polished). This reduces their applicability and increas the associated cost. Second, for electrodes below approximately 10 microns in diameter electrodes often exhibit significantly larger capacitances than anticipated on the basis of their geometric size [13]. For an ideal microelectrode, the capacitance is dominated by the electroactive interface. In this case the capacitative current should be virtually unmeasurable via cyclic voltammetry at the commonly used experimental scan rates $\left(\sim 0.1 \mathrm{~V} \mathrm{~s}^{-1}\right)$. The origin of this often encountered excess capacitance is reportedly predominantly from either stray capacitances at the electrode [14] or from poor sealing between the surrounding insulator and the electrode material [15]. Importantly, this problem of 'apparently' large capacitances occurs for not just homemade but also for the extensively used commercial microdisc electrodes. It should also be commented that microelectrode arrays - depending on their design - may be even more susceptible to the influence of stray- capacitances further limiting their sensitivity [16]. Therefore, exploring an electrode with an alternative geometry (microcylinder) which may be superior over the most commonly used geometry (microdisc) is of interest and is investigated in the present work.

Recent work has demonstrated how carbon fibre microcylinder electrodes are of practical use for the detection of low concentrations of nanoparticles $[17,18]$. Platinum microcyclinders have also recently been used for oxygen detection and in a microfuel cell $[19,20]$. The present paper first, experimentally demonstrates that the apparent capacitance per unit area of a carbon fibre microcylinder electrode is distinctly smaller than that found for a commercial glass sealed carbon microdisc electrodes. The reasons for the low apparent capacitance of the carbon fibre microcylinder electrodes are explored. Second, the article continues by demonstrating practically how the electroanalytical limit of detection of solution phase oxygen is significantly improved using a carbon fibre microcylinder electrode. This improvement is seen despite the greater Faradaic current density at the microdisc electrode as compared to the microcylinder. The carbon microcylinder electrode is finally used to demonstrate that after degassing of a solution by nitrogen purging tens of micromolar oxygen remains in a typical electrochemical cell.

\section{Experimental}

\subsection{Chemicals}

Hexamineruthenium chloride $\left(\mathrm{Ru}\left(\mathrm{NH}_{3}\right)_{6} \mathrm{Cl}_{3}\right)$, potassium chloride $(\mathrm{KCl})$, sodium phosphate dibasic $\left(\mathrm{Na}_{2} \mathrm{HPO}_{4}\right)$ and sodium phosphate monobasic $\left(\mathrm{NaH}_{2} \mathrm{PO}_{4}\right)$ were purchased from Aldrich (Gillingham, UK) at the highest available grade $(99.0 \%)$ and used as received. High purity oxygen and nitrogen were obtained from BOC (Guildford, UK). All solutions were prepared with ultrapure water (Millipore UHQ, Vivendi, UK) with a resistivity of not less than $18.2 \mathrm{M} \Omega \mathrm{cm}$ at $25^{\circ} \mathrm{C}$. Phosphate buffer (PB) was prepared by mixing $100 \mathrm{mM}$ aliquots of $\mathrm{Na}_{2} \mathrm{HPO}_{4}$ and $\mathrm{NaH}_{2} \mathrm{PO}_{4}$ and adjusting the $\mathrm{pH}$ to 7.2. The measurement of $\mathrm{pH}$ was performed using a Hannah $\mathrm{pH}$ $213 \mathrm{pH}$ meter. Prior to experiments, working solutions were purged with nitrogen or oxygen for $15 \mathrm{~min}$. All experiments were carried out in a thermostated water bath at a temperature of $25.0 \pm 0.1{ }^{\circ} \mathrm{C}$.

\subsection{Fabrication and characterization of carbon microcylinder electrodes}




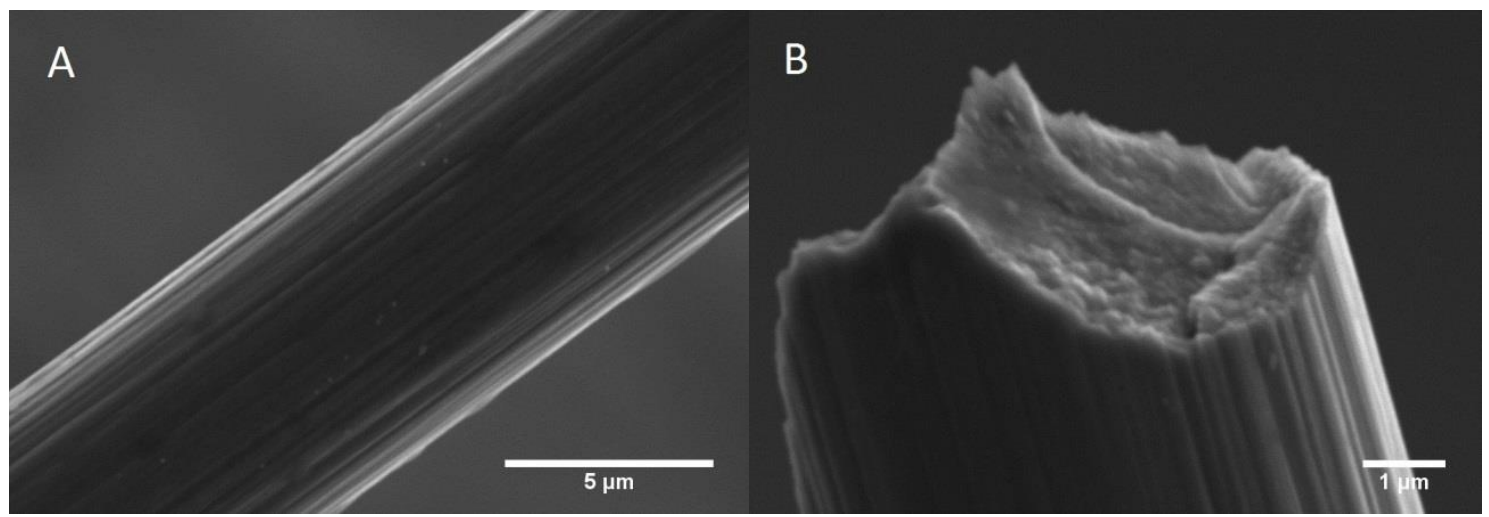

Fig. 1. SEM images of carbon fibre used for microcylinder electrode preparation: side view of the fibre (A), cross-section of the fibre (B).

Prior to the fabrication procedure, carbon fibre wire characterization was performed by scanning electron microscopy (SEM) using a Zeiss Evo Scanning Electron Microscope with an accelerating voltage of $5 \mathrm{kV}$. Sample preparation involved dispersing pre-cut fibres on a conductive copper substrate in order to obtain a range of orientations. As can be seen, the electrode surface is relatively smooth (Fig. 1A). The bulk consists of fused individual strands (Fig. 1B). The average wire radius of $3.51 \pm 0.01 \mu \mathrm{m}$ was determined from the SEM images.

Having characterized the carbon fibre, the cylindrical microelectrodes were fabricated in accordance with method reported previously in literature [17]. In brief, a carbon fibre with the diameter of $7.0 \mu \mathrm{m}$ (Goodfellow Cambridge Ltd.) was connected to a conducting metal wire using silver epoxy (RS Components Ltd.) conductive adhesive. The adhesive was heated in an oven at $60{ }^{\circ} \mathrm{C}$ for $15 \mathrm{~min}$. Subsequently, the wire was placed inside the plastic pipette tip so that only carbon fibre was extended. In order to prevent electrical leakage, the plastic tip was sealed with cyanoacrylate adhesive and the carbon fibre was cut to obtain a length of approximately $1 \mathrm{~mm}$.

The accurate length of the manufactured microcylinder electrode was determined by measuring the quasi steadystate current at various scan rates of $1.0 \mathrm{mM}$ ruthenium (III) hexamine in $100 \mathrm{mM} \mathrm{KCl}$ as a supporting electrolyte at $298 \mathrm{~K}$ and fitting the length as a fitting parameter according to the theoretical predictions (see section 3.1 for further details).

\subsection{Apparatus}

Cyclic voltammetric measurements were performed in a glass electrochemical cell $(25 \mathrm{~mL})$ inside a Faraday cage. A $\mu$ Autolab III potentiostat (Metrohm Autolab B.V., The Netherlands) was used and controlled by NOVA 1.10 electrochemical software. A standard three-electrode configuration was used. This employed a saturated calomel reference electrode (SCE), a platinum microdisc electrode as a counter electrode and either a fabricated carbon-wire microcylinder electrode or a commercial carbon microdisc as the working electrode. The carbon microdisc electrode was consecutively polished before each experiment using 1.0, 0.3 and $0.01 \mu \mathrm{m}$ aluminawater slurry on soft lapping pads (Buehler, Illinois). The electrochemical cell was covered with a plastic lid and parafilm to minimize the penetration of oxygen to the solution after degassing. Subsequent numerical data analysis was performed using software Mathematica 10.4 .

\section{Results and Discussion}

This section starts with the characterisation of the geometrical surface area of commercial carbon microdisc and fabricated carbon microcylinder electrodes. Next, the apparent double layer capacitance per geometric area of the two electrode types is evaluated. Having characterized the electrode response, the oxygen reduction reaction is studied by cyclic voltammetry in a range of oxygen saturation conditions (degassed, airequilibrated and full-oxygen saturation). The relative contribution of the capacitance and the Faradaic components is discussed. A practical limit of detection is established for the commercial microdisc electrodes and the home-built microcylinder electrode. Finally, it is 
shown that tens of $\mu \mathrm{M}$ of oxygen remain present in the electrochemical cell. These levels are unobservable by the commercial microdisc electrodes after conventional degassing with nitrogen. But they are detectable with microcylinder electrodes.
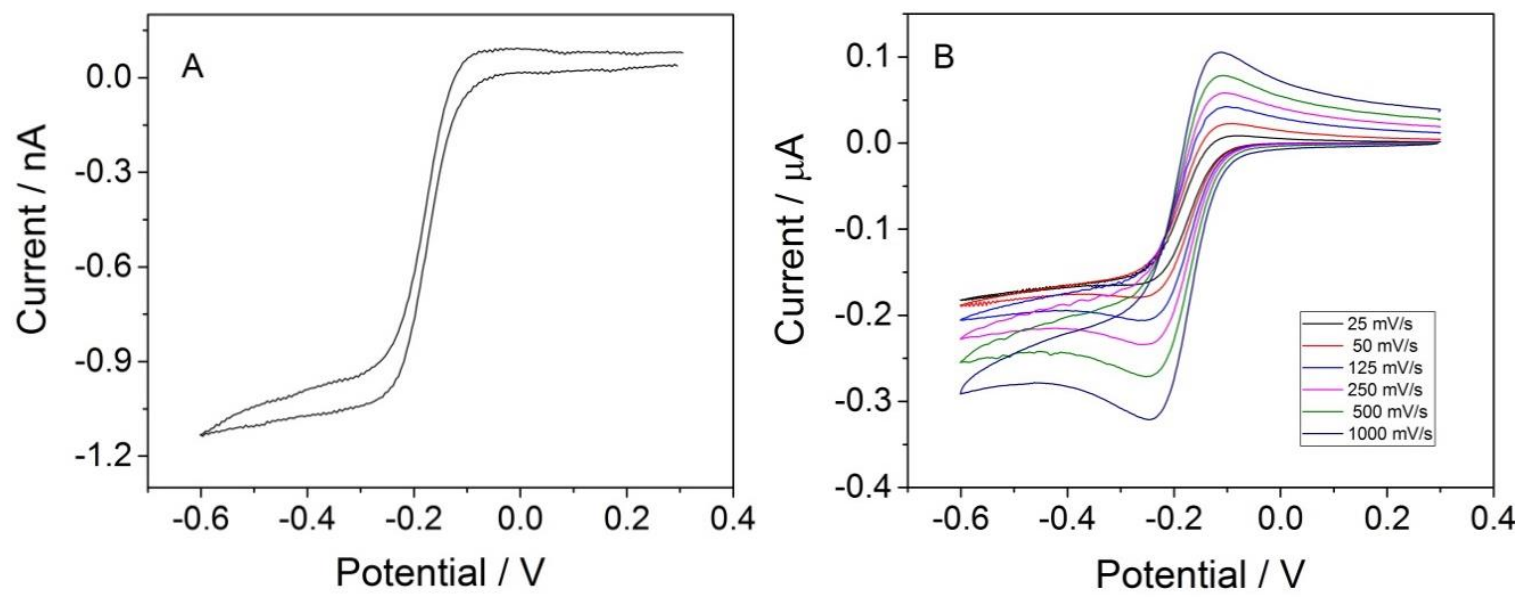

Fig. 2. Cyclic voltammograms of $1.0 \mathrm{mM}$ ruthenium(III) hexamine in $100 \mathrm{mM} \mathrm{KCl}$ as a supporting electrolyte at varying scan rates at $100 \mathrm{mV} \mathrm{s}^{-1}$ using commercial carbon microdisc electrode (A) and fabricated carbon microcylinder electrode (B). Potentials are shown versus an SCE electrode.

\subsection{Electrode area determination}

\subsubsection{Microdisc electrode area determination}

The electrodes used in the present work were first characterised electrochemically by cyclic voltammetry. The geometric area accessible to diffusing analytes was determined. This was used for the subsequent data analysis. This determined the apparent electrode capacitance per unit area. The radii of the commercial carbon microdisc electrodes were calculated by measuring the steady-state limiting current $\left(I_{s s}\right)$ using the outer sphere redox probe ruthenium (III) hexamine [21] (Eq. 1). A typical cyclic voltammogram of $1.0 \mathrm{mM}$ ruthenium(III) hexamine in $100 \mathrm{mM} \mathrm{KCl}$ at a scan rate of $100 \mathrm{mV} \mathrm{s}^{-1}$ is depicted in Fig. 2A. A clear steady-state response was observed. This is consistent with a diffusionally limited current. The following fitting parameters were used to calculate the radius of the microdisc in accordance with Eq. $2: \mathrm{D}=8.43 \times 10^{-10} \mathrm{~m}^{2}$ $\mathrm{s}^{-1}$ [22], $\mathrm{C}=1 \mathrm{~mol} \mathrm{~m}^{-3}, \mathrm{n}=1$ and $\mathrm{F}=96485.3 \mathrm{C} \mathrm{mol}^{-1} . \mathrm{As}$ a result, the radius of the carbon microdisc electrode was calculated as $3.35 \pm 0.03 \mu \mathrm{m}$. This shows a total geometric area of $3.14 \times 10^{-7} \mathrm{~cm}^{2}$. This procedure was repeated for all the microdisc electrodes studied.

\subsubsection{Microcylinder electrode area determination}

In order to calculate the geometric area of a microcylinder, an accurate length of the carbon wire is required (the radius was determined previously using SEM imaging, see section 2.2). The length of the microcylinder electrode can be determined by measuring the quasi steady-state currents from a diffusing species at a range of scan rates. Subsequently fitting to the theoretically predicted currents used the length as a fitting parameter. The theoretical currents can be predicted for varying scan rates by the equation derived by Aoki et al. This assumes negligible edge effects [23]:

$\frac{I_{p}}{2 \pi n F c D l}=0.446 p+0.335 p^{0.15}$ with $p=\left(\frac{n F r^{2} v}{R T D}\right)^{0.5}$ 

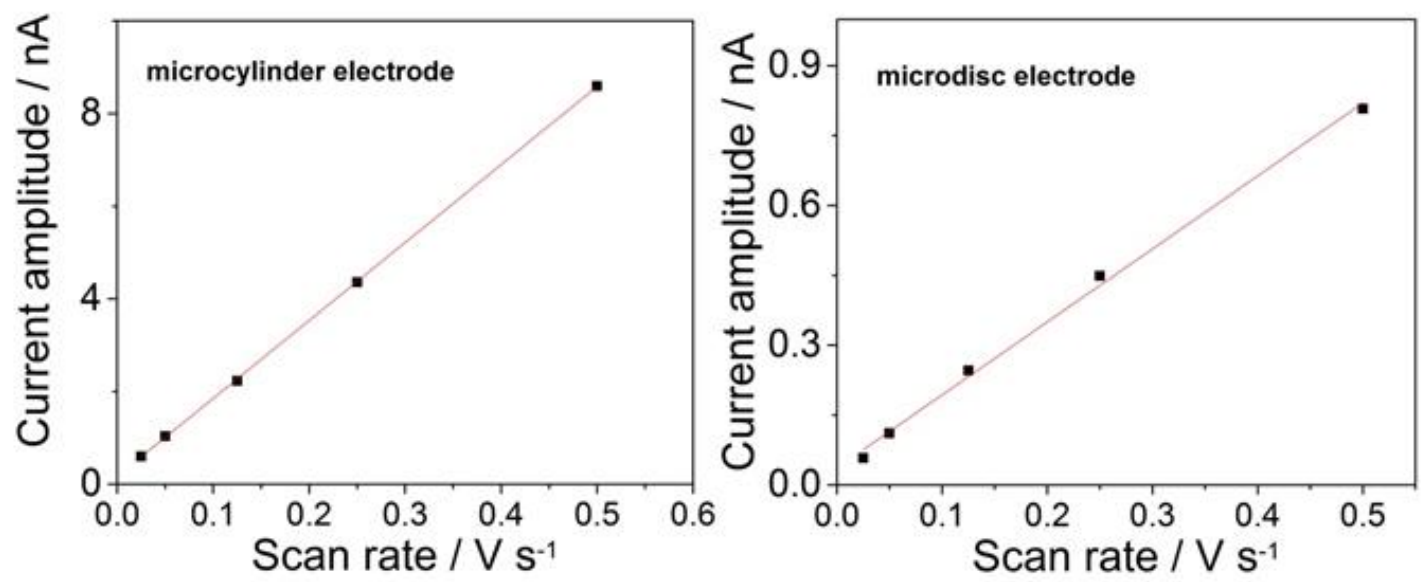

Fig. 3: Current amplitude in the capacitance region as a function of scan rate for the carbon microcylinder (left) and microdisc (right) electrodes.

Table 1. Specific apparent capacitances for a set of fabricated carbon microcylinder and carbon microdisc electrodes

\begin{tabular}{ccccc}
\hline \multirow{2}{*}{ Electrode } & \multicolumn{4}{c}{ Capacitance $\left(\mu \mathrm{F} \mathrm{cm}^{-2}\right)$} \\
& Repeat 1 & Repeat 2 & Repeat 3 & Average \\
\hline W1 & 49.71 & 42.3 & 52.3 & $48.1 \pm 5.2$ \\
W2 & 100 & 99.7 & 99.5 & $99.7 \pm 0.3$ \\
W3 & 52.4 & 47.9 & 44.8 & $48.3 \pm 3.8$ \\
W4 & 46.0 & 42.7 & 41.4 & $43.3 \pm 2.4$ \\
W5 & 48.0 & 46.8 & 44.8 & $46.5 \pm 1.6$ \\
D1 & 675 & 2265 & 1086 & $1342 \pm 825$ \\
D2 & 3386 & 2068 & 1564 & $2339 \pm 940$ \\
D3 & 1511 & 2158 & 2915 & $2195 \pm 702$ \\
D4 & 1923 & 1658 & 2518 & $2033 \pm 440$ \\
\hline W $=$ carbon
\end{tabular}

$\mathrm{W}=$ carbon microcylinder electrode, $\mathrm{D}=$ carbon microdisc electrode

Here $I_{p}$ is theoretical peak current, $\mathrm{n}$ is the number of electrons transferred, $F$ is Faraday constant $(96485.3 \mathrm{C}$ $\left.\mathrm{mol}^{-1}\right), c$ is the analyte concentration $\left(\mathrm{mol} \mathrm{m}^{-3}\right), D$ is the diffusion coefficient of the analyte $\left(\mathrm{m}^{2} \mathrm{~s}^{-1}\right), l$ is the length of the wire, $r$ is the radius of the electrode, $v$ is the experimental scan rate $\left(\mathrm{V} \mathrm{s}^{-1}\right), T$ is the temperature $(\mathrm{K})$ and $R$ is the gas constant $\left(8.314 \mathrm{~J} \mathrm{~K}^{-1} \mathrm{~mol}^{-1}\right)$. In particular, the length of the fabricated carbon-wire microelectrode can be estimated by plotting the experimentally observed peak current against the scan rate. Least squares fitting was used to determine the length in accordance with the theoretical prediction. Typical cyclic voltammograms of $1.0 \mathrm{mM}$ ruthenium(III) hexamine in $100 \mathrm{mM} \mathrm{KCl}$ were recorded on a carbon microcylinder electrode at a range of scan rates from 10to $1000 \mathrm{mV} \mathrm{s}-1$ cathodically from $0.30 \mathrm{~V}$ to $-0.60 \mathrm{~V}$ (vs. SCE) and back (Fig 2B). Using the radius of the electrode obtained from SEM measurement, the length of carbon fibre was estimated to be $0.97 \pm 0.11 \mathrm{~mm}$, yielding a geometric area of $2.14 \times 10-4 \mathrm{~cm} 2$. This procedure was repeated for all microcylinder electrodes fabricated in this study.

\subsection{Capacitance measurements and geometrical considerations}

Having determined the geometric area of the two types of electrodes it is possible to determine the specific apparent capacitance per geometric area $(\mu \mathrm{F} \mathrm{cm}-2)$ (Note that surface roughness is not taken into account as the object of the present study is in analytical applications and not the fundamental study of the double layer. Thus the geometric area accessible to a diffusing analyte is 
pertinent). The practical limit of analyte detection is determined by the Faradaic as well as capacitative current components. In general, the voltammetric measurement gives the sum of capacitive and Faradaic current contributions. Ideally the capacitive current scales linearly with the area of an electrode whilst the Faradaic current increases linearly with the electrode radius for a microelectrode (disc or wire).

As a result it is critical to consider the capacitive contribution to the observed current response as it may mask the Faradaic signal of interest. A Faradaic reaction involves electrons crossing the electrode/solution boundary. The experimentally observed current in the potential region where no Faradaic reaction occurs because of thermodynamic and kinetic limitations is related to the capacitance in accordance with Eq. 1. Experiments were conducted on four microdisc and five microcylinder electrodes.

For each electrode studied, cyclic voltammograms were recorded in PB buffer $\mathrm{pH} 7.2$ at varying scan rates $(25-$ $500 \mathrm{mV} \mathrm{s}^{-1}$ ) and the current amplitude at the potential of $0 \mathrm{~V}$ (vs SCE) (see Supporting information, SI, Section 1) was plotted as a function of scan rate (Fig.3). Subsequently, the apparent capacitance normalized for the electrode geometric area was calculated from the slope of this dependence (Table 1).

According to Table 1, average apparent capacitance of the commercial carbon microdisc electrodes is found to be $1977 \pm 441 \mu \mathrm{F} \mathrm{cm} ~^{-2}$, while for the microcylinder electrodes $57 \pm 24 \mu \mathrm{F} \mathrm{cm}^{-2}$. For an ideal electrode where the capacitance is solely due to double layer effects a value of ca $20 \mu \mathrm{F} \mathrm{cm}{ }^{-2}$ is expected. [6] The slight increase of capacitance in the case of microcylinder electrodes. In comparison, the large capacitances of the commercial microdisc electrodes reflects the polishing procedure and variability of the microdiscs used in the work. In particular, it is evident that for the microcylinder electrode the specific capacitance per area is approximately lower when compared to microdisc by a factor of 35 ! This is a large unexpected difference but is consistently observed for the commercial microelectrodes (see SI, Section 2 which shows cyclic voltammogram supplied by the manufacturer, which clearly also indicates an apparent capacitance of $2381 \mu \mathrm{F} \mathrm{cm} \mathrm{cm}^{-2}$ ). As both type of electrodes are nominally of the same material (carbon fibre) one would not expect the true specific capacitance to differ so significantly if it solely reflects the electrode-solution interface. The explanation for the increased capacitance having no origin in charging of double-layer has been previously suggested as a combination of two factors: stray capacitances at the electrode [14] and a potentially poor sealing between the surrounding insulator and the electrode material [15].

In order to understand the practical limit of detection we also need to consider the difference in geometries for the two types of electrodes used and the effect on the observed mass transport. In the case of microdisc electrodes, diffusion generally occurs in different ways. First at very short times the mass transport process is dominated by planar diffusion. Second, at longer timescales radial diffusion becomes dominant giving very high rates of mass transport. This results in an observation of a steady-state current [24,25]. The efficiency of the radial diffusion is determined by the size of an electrode. It is more dominant as the size of an electrode decreases. There is an absence of a back-peak in the cyclic voltammogram for a microdisc electrode. This is the electrolysis product leaves the diffusion layer at an enhanced rate.

In comparison, the microcylinder electrodes used in the present work have microscopic radii (of several micrometres) but a macroscopic length (of the order of millimetres) As a result of a macroscopic length, the radial diffusion is not as efficient in comparison to a microdisc electrode, and a peaked voltammogram response is observed. A back peak is clearly present (see Fig. 2 B) which is indicative of a lower diffusion efficiency and consequently a lower rate of mass transport [24].

In accordance with these theoretical arguments in terms of the current density, a microdisc electrode is clearly expected to be superior and an enhanced sensitivity towards analytes might be expected. However, despite the current density being lower for the microcylinder in comparison to microdisc electrode, as the surface area of the microcylinder electrodes is generally much larger and in combination with a significantly lower specific capacitance the practical limit of detection may be anticipated to be lower for such electrodes. In the next section, we test this hypothesis.

\subsection{Oxygen reduction}

Having characterized the area of the electrodes and determined the apparent capacitance and discussed the geometrical considerations for mass transport, the oxygen reduction reaction was studied next on both fabricated microcylinder electrodes and commercial carbon microdisc electrodes. Oxygen was chosen due to its omnipresence, significant reactivity and a wide range of important technological applications [26,27]. 

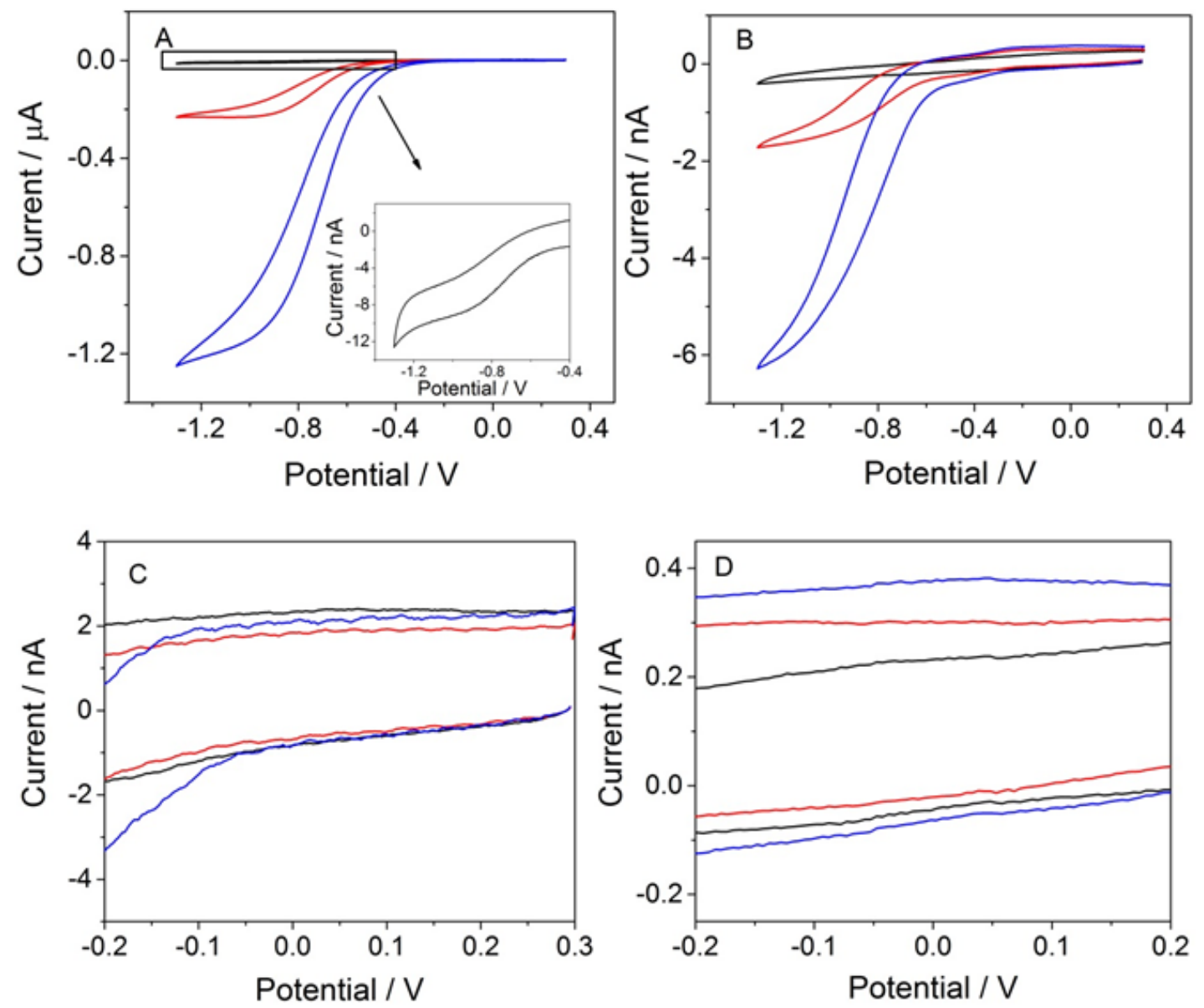

Fig. 4: Cyclic voltammograms of PB pH 7.2 using either a fabricated carbon microcylinder (A) or a commercial microdisc electrode (B) and the zoom of capacitance region for carbon microcylinder (C) and commercial microdisc electrode (D). Black line - $\mathrm{N}_{2}$ degassed solution, red line - air-equilibrated solution, blue line - solution purged with $\mathrm{O}_{2}$ at the scan rate of $125 \mathrm{mV} \mathrm{s}$. Potentials are shown versus an SCE electrode.

Oxygen reduction reaction (ORR) in acidic media is a highly complex reaction. Currently the accepted mechanism for the ORR on carbon electrodes involves an overall transfer of two electrons. Hydrogen peroxide species are formed [28-30]. The process can be summarized by the following reaction:

$$
\mathrm{O}_{2}+2 \mathrm{e}^{-}+2 \mathrm{H}^{+} \rightarrow \mathrm{H}_{2} \mathrm{O}_{2}
$$

For each electrode studied, first, the response of airequilibrated solution was investigated. Subsequently, the solution was thoroughly degassed with nitrogen for 15 min and purged with oxygen for $15 \mathrm{~min}$. After each step, the cyclic voltammetric response was recorded at a range of scan rates from 25 to $500 \mathrm{mV} \mathrm{s}^{-1}$ (see SI, Section 3). Fig. 4 shows typical voltammetric responses of fabricated microcylinder and microdisc electrodes towards airequilibrated (red line), degassed (black line) and oxygen saturated solutions (blue line) in the presence of $\mathrm{PB} \mathrm{pH}$ 7.2 at the scan rate of $125 \mathrm{mV} \mathrm{s}^{-1}$. The cyclic voltammetric scans were performed in a potential window from $+0.30 \mathrm{~V}$ to $-1.30 \mathrm{~V}$ vs SCE. In the case of carbon microcylinder electrodes, a wave was exhibited at $-0.90 \mathrm{~V}$. This corresponds to the reduction of oxygen. Significantly this was present even after degassing with nitrogen in the nominal absence of oxygen. As for the microdisc electrodes, the oxygen reduction wave was observed for the $\mathrm{O} 2$ purged and air-equilibrated solution. Importantly, the reduction wave was absent after degassing. This observation can be explained by the large specific capacitance of the microdisc electrode (see Table 1). The Faradaic response of the ORR is completely 'hidden' by the large capacitive component leading to an apparent non-observation of the dissolved oxygen. The absence of a wave corresponding to the reduction of oxygen does not indicate the solution is oxygen-free. It is important to investigate the amount of oxygen remaining in the solution after the degassing procedure. In the next step, the data obtained using a carbon microcylinder electrode was further evaluated by plotting the current at 
the potential of $-1.3 \mathrm{~V}$ (vs SCE) as a function of the scan rate (SI, Section 5) in order to estimate the residual oxygen concentration. The solubility of oxygen in solution is proportional to the partial pressure of the gas over the solution. It can be calculated by applying Henry's law. Assuming the ratio of steady state currents reflects the theoretical ratio of partial oxygen pressures for the air-equilibrated and oxygen-saturated solutions we can compare the experimentally observed ratio with an expected value derived from the application of the Henry's law in accordance with Equation 5:

$$
\begin{aligned}
& \frac{P_{O_{2} \text { air }}}{P_{O_{2} \text { sat }}}=\frac{I_{O_{2} \text { air }}}{I_{O_{2} \text { sat }}}=0.21 \\
& \frac{P_{O_{2} \text { air }}}{P_{O_{2} \text { sat }}}=\frac{I_{O_{2} \text { air }}}{I_{O_{2} \text { sat }}}=0.21
\end{aligned}
$$

where $\mathrm{P}_{\mathrm{O} 2}$ air is the partial pressure of oxygen in the air atmosphere, $\mathrm{P}_{\mathrm{O} 2 \text { sat }}$ is the partial pressure of oxygen in the oxygen atmosphere $\left(\mathrm{P}_{\mathrm{O} 2 \mathrm{sat}}=1 \mathrm{~atm}\right)$, IO2air is the current recorded at a potential of $-1.3 \mathrm{~V}$ vs SCE in the air saturated solution, $\mathrm{I}_{\mathrm{O} 2 \mathrm{sat}}$ is the current recorded at a potential of $-1.3 \mathrm{~V}$ vs SCE in the oxygen saturated solution. The expected ratio from the application of Henry's law is 0.21 . For a microcylinder electrode the ratio of IO2air to IO2sat was determined experimentally to be 0.20 in excellent agreement with the expected value. For the microdisc electrode the ratio of $\mathrm{I}_{\mathrm{O} 2 \mathrm{air}}$ to $\mathrm{I}_{\mathrm{O} 2 \mathrm{sat}}$ was determined as 0.28. The discrepancy between the expected ratio from the Henry's law and the ratio experimentally obtained for microdisc electrodes could arise due to the imperfect seal of the microdisc electrodes and microfractures leading to an observation of a higher ratio resulting from liquid layers trapped between the electrode and the insulating sheath. Taking into account the oxygen concentration in fully saturated solution to be $1.26 \mathrm{mM}$ [31], the residual concentration of oxygen in a nitrogen-purged solution using a microcylinder electrode was estimated to be $12.6 \mu \mathrm{M}$.

Such concentrations of molecular oxygen may have significant effect on low analyte concentration experiments. First, oxygen is known to react with many species. Second, the presence of oxygen may lead to fluorescence-quenching of various fluorophores and as result can have a detrimental effect on spectroelectrochemical studies [32]. Given the results revealed above, it would be unwise to regard the absence of oxygen signals measured at a microdisc electrode to signify the absence of oxygen. In order to reduce the levels of dissolved oxygen an alternative to outgassing may be warranted. Ultra-low oxygen-containing solutions can be achieved by using sodium sulfite and copper sulfate as a catalyst to chemically bind oxygen. However, this approach may lead to other complications as sodium sulphite is electrochemically active [33]. Degassing can be effectively achieved via photochemical reaction of oxygen with a high concentration of formic acid radical under ultra-violet irradiation (wavelength $<260 \mathrm{~nm}$ ) [34,35].

\section{Conclusions}

In this paper we have demonstrated three important considerations for accurate electroanalytical measurements. First, a reduced geometric area does not necessarily lead to practically low capacitances due to factors such as surface roughness, poor sealing and potential microfractures leading to a significant variation in experimentally observed capacitance. Comparing the specific apparent capacitances of working electrodes, the capacitance of fabricated carbon microcylinder electrode was found to be lower by a factor of 35 despite the fact that both type of electrodes were made of nominally the same material. As a consequence of the reduced capacitance carbon microcylinder electrodes were experimentally proven to be superior in terms of analytical sensitivity over commercial carbon microdisc electrodes. This was despite the less efficient mass transport and the reduced Faradaic

current density. Microcylinder electrodes are inherently cheap and easy to fabricate [17]. They are also disposable which will likely facilitate their use in sensing applications. As a result of the reduced specific apparent capacitance of the microcylinder electrodes cyclic voltammetry revealed the presence of micromolar (ca 10 $\mu \mathrm{M}$ ) oxygen remaining in degassed aqueous solutions, which is unobservable using commercial carbon microdisc electrodes. This observation further highlights the challenges of efficient degassing and could have significant implications for spectroelectrochemistry. In the latter even the presence of micromolar concentrations of oxygen may lead to significant fluorescence quenching.

\section{Acknowledgements}

This work was supported by the Grant Agency of the Slovak Republic (Grant No. 1/0489/16), Grant scheme for Support of Young Researchers and Excellent Teams of Young Researchers. KC acknowledges financial support by the National Scholarship Programme of the Slovak Republic and Tatrabanka Foundation for supporting her research stay in Oxford. SS, CBM and 
RGC acknowledge partial funding from the European Research Council under the European Union's Seventh Framework Programme (FP/2007-2013)/ERC Grant Agreement no. [320403].

\section{References}

[1] R. Payne, J. Phys. Chem. 1965, 70, 204-212.

[2] R. Payne, J. Phys. Chem. 1965, 69, 4113-4123.

[3] I. M. Barclay, J. A. V. Butler, Trans. Faraday. Soc. 1940, $35,128-136$.

[4] D. C. Grahame, J. Am. Chem. Soc. 1949, 71, 2975-2978.

[5] H. Gerischer, R. McIntyre, D. Scherson, W. Storck, J. Phys. Chem. 1987, 91, 1930-1935.

[6] A. J. Bard, L. R. Faulkner, Electrochemical Methods, Wiley, New York, 1980.

[7] T. Pajkossy, D. M. Kolb, Electrochem. Commun. 2007, 9, 1171-1174.

[8] R. M. Wightman, Anal. Chem. 1981, 53, 1125-1134.

[9] J. W. Bixler, A. M. Bond, P. A. Lay, W. Thormann, P. van den Bosch, Anal. Chim. Acta 1986, 187, 67-77.

[10] R. G. Compton, C. E. Banks, Understanding Voltammetry, Imperial College Press, London, 2011.

[11] S. Fletcher, M. D. Horne, Electrochem. Commun. 1999, 1, 502-512.

[12] A. D. Lançon, R. Kataky, D. Wood, A. J. Gallant, Electrochem. Commun. 2011, 13, 414-417.

[13] D. O. Wipf, A. C. Michael, R. M. Wightman, J. Electroanal. Chem. 1989, 269, 15-25.

[14] J. Heinze, Angew. Chem. Int. Ed. Engl. 1993, 32, 12681288.

[15] K. R. Wehmeyer, R. M. Wightman, J. Electroanal. Chem. 1985, 196, 417-421.

[16] S. G. Weber, Anal. Chem. 1989, 61, 295-302.

[17] J. Ellison, C. Batchelor-McAuley, K. Tschulik, R. G. Compton, Sens. Actuator B-Chem. 2014, 200, 47-52.

[18] C. Batchelor-McAuley, J. Ellison, K. Tschulik, P. Hurst, R. Boldt, R. G. Compton, Analyst, 2015, 140, 5048-5054

[19] S. T. Singleton, J. J. O’Dea, J. Osteryoung, Anal. Chem. 1989, 61, 1211-1215.

[20] J. Weber, A. J. Wain, G. A. Attard, F. Marken, Electroanalysis, 2016, 28, in press.

[21] L. Xiong, C. Batchelor-McAuley, K. R. Ward, C. Downing, R. S. Hartshorne, N. S. Lawrence, R. G. Compton, J. Electroanal. Chem. 2011, 661, 144-14.

[22] Y. Wang, J. G. Limon-Petersen, R. G. Compton, J. Electroanal. Chem. 2011, 652, 13-17.
[23] K. Aoki, K. Honda, K. Tokuda, H. Matsuda, J. Electroanal. Chem. Interfacial Electrochem. 1985, 187, 267-279.

[24] R. J. Forster, Chem. Soc. Rev. 1994, 23, 289-297.

[25] S. Pons, M. Fleischmann, Anal. Chem. 1987, 59, 13911399.

[26] I. Katsounaros, S. Cherevko, A. R. Zeredjanin, K. J. J. Mayrhofer, Angew. Chem. 2014, 53, 102-121.

[27] D. T. Sawyer, L. V. Interrante, J. Electroanal. Chem. 1961, 2, 310-327

[28] R. Nissim, R. G. Compton, ChemElectroChem 2014, 1, 763-771.

[29] H. H. Yang, R. L. McCreery, J. Electrochem. Soc. 2000, $147,3420-3428$.

[30] B. Šljukić, C. E. Banks, R. G. Compton, J. Iran. Chem. Soc. 2005, 2, 1-25.

[31] K. Shimizu, L. Sepunaru, R. G. Compton, Chem. Sci. 2016, 7, 3364-3369.

[32] J. R. Lakowicz, G. Weber, Biochemistry 1973, 12, 41614170 .

[33] K. A. Kobe, W. L. Gooding, Ind. Eng. Chem. 1935, 27, 331-333.

[34] T. Matsuura, J. M. Smith, AIChE J. 1970, 16, 1064-1071.

[35] M. Bideau, B. Claudel, M. Otterbein, J. Photochem. 1980, 14, 291-302. 